\title{
Artificial neural network (ANN) prediction of compressive strength of VARTM processed polymer composites
}

\author{
A. Tuğrul Seyhan ${ }^{\text {a }}$, Gökmen Tayfur ${ }^{b}$, Murat Karakurt ${ }^{a}$, Metin Tanoğlu ${ }^{\text {a,* }}$ \\ a Department of Mechanical Engineering, Izmir Institute of Technology, Gülbahçe Kampüs 35437, Urla / Izmir, Turkey \\ ${ }^{\mathrm{b}}$ Department of Civil Engineering, Izmir Institute of Technology, Gülbahçe Kampüs 35437, Urla / İzmir, Turkey
}

Received 26 May 2004; received in revised form 21 September 2004; accepted 14 November 2004

\begin{abstract}
A three layer feed forward artificial neural network (ANN) model having three input neurons, one output neuron and two hidden neurons was developed to predict the ply-lay up compressive strength of VARTM processed E-glass/ polyester composites. The composites were manufactured using fabric preforms consolidated with 0,3 and $6 \mathrm{wt} . \%$ of thermoplastic binder. The learning of ANN was accomplished by a backpropagation algorithm. A good agreement between the measured and the predicted values was obtained. Testing of the model was done within low average error levels of $3.28 \%$. Furthermore, the predictions of ANN model were compared with those obtained from a multi-linear regression (MLR) model. It was found that ANN model has better predictions than MLR model for the experimental data. Also, the ANN model was subjected to a sensitivity analysis to obtain its response. As a result, the ANN model was found to have an ability to yield a desired level of ply-lay up compressive strength values for the composites processed with the addition of the thermoplastic binder.
\end{abstract}

(c) 2004 Elsevier B.V. All rights reserved.

PACS: 81-20; 81.70.B

Keywords: Compressive strength; Artificial neural network (ANN); Polymer composites; Preforming binder; Multi-linear regression (MLR)

\footnotetext{
* Corresponding author. Tel.: +90 232750 6597; fax: +90 232750 6505/498 6500.

E-mail address: metintanoglu@iyte.edu.tr (M. Tanoğlu).
}

\section{Introduction}

Vacuum-assisted resin transfer molding (VARTM), a derivative of the liquid molding (LM) process, has been widely employed to manufacture advanced composite structures especially 
for defense and civil engineering applications [1,2]. VARTM is typically a three-step process including lay-up of a fiber performs on a tool, infusion of the preform with a liquid resin, and the cure of infused resin within the preform. Fiber volume fraction $\left(V_{\mathrm{f}}\right)$ is one of the critical property for the polymer composites and it may have some significant effects on the composite mechanical properties. The degree of the compaction of the fiber preform is known to have some significant effects on the fiber volume fraction, porosity formation and resin flow characteristic within the reinforcement [3]. Therefore, understanding the effects of preform compaction and mutually fiber volume fraction on the composite mechanical behavior is essential. The recent technique to consolidate the fiber performs is to use powdered thermoplastic binders between the adjacent plies to compact them briefly $[1,4]$. Binder-coated plies with various binder concentration can be stacked together under application of heat and pressure. As the thickness of the fabric preform reduces, in general, the fiber volume fraction increases. In the previous work [5], it was concluded that compressive stress-strain behavior of the E-glass/polyester composites loaded along the ply-lay up and in-plane direction were considerably affected by the preforming binder. Preform compaction experiments revealed that the highest compaction can be obtained with $3 \mathrm{wt} . \%$ of the binder and the further increase of binder concentration resulted in increasing of the preform thickness. It was also revealed that the composites composed of fabric preforms with $3 \mathrm{wt} . \%$ of binder exhibited the highest ply-lay up and in-plane compressive strength and modulus than those with 0 and $6 \mathrm{wt} . \%$ of binder.

In addition, fiber preform compaction during VARTM process may not be uniform across the length of the part, as the resin fills the preform from one side by the means of vacuum pressure $[5,4]$. Thus, the fiber volume fraction and resin permeability may not be constant and varies through the part. This may result in considerable thickness variations and non-uniform mechanical properties through the composite part [5,2]. The understanding of the influence of several factors in VARTM that affect the overall mechanical properties such as strength of the composites may be important.
These factors may include the thermoplastic binder content (\%wt.), fiber preform thickness prior to VARTM processing and the composite fiber volume fraction. Each of them may have varying degrees of effect on the overall strength of the composite parts [1]. However, an analytical model to describe the effects of such factors together on the strength can be very complex $[4,6]$. Therefore, an artificial neural network (ANN) approach can be used as a powerful tool in modeling the effects of a various parameters on ply-lay up compressive strength of the composites. A certain amount of experimental data is necessary to develop a wellperforming neural network, including its architecture, training functions and training algorithms $[7,8]$. The greatest advantage of ANN is its ability to model complex non-linear, multi-dimensional function relationships without any prior assumptions about the nature of the relationships $[9,6]$. As an example, Zhang et al. [8] developed an ANN model to predict the dynamic mechanical properties of PTFE-based composites with various short carbon fiber contents. They found that the number of training data set is an important parameter in ANN predictive quality. Therefore, an one-output neural network is suggested to be use initially for high predictive quality before a sufficient database is available. Wear performance of polyethylene (PE), polyurethane (PUR), and an epoxy modified by hygrothermally decomposed polyurethane (EP-PUR) was also predicted by the same authors using an ANN model [6]. They concluded that a well-trained ANN model is the key to design and analysis structure-property relations of the polymer composites.

In the present study, an ANN approach was employed to predict the effects of the thermoplastic binder concentration $\left(C_{\mathrm{b}}\right)$, fabric preform thickness prior to process $(t)$ and composite fiber volume fraction $\left(V_{\mathrm{f}}\right)$ on the ply-lay up compressive strength of VARTM processed E-glass fiber reinforced polyester composites. The ANNs software was trained and tested with sets of experimental data consisting of $C_{\mathrm{b}}, t$, and $V_{\mathrm{f}}$ as input and composite ply-lay up compressive strength as output. Furthermore, the predictions of the ANN model were compared to those with a multi-linear regression (MLR) model. 


\section{Experimental}

Experimental procedure was described in detail in the previous work [7]. In brief, composite parts were fabricated using E-glass fabrics and Camelyaf 266 thermosetting polyester resins both purchased from Cam Elyaf of Corp of Turkey. Cobalt naphthenate (CoNAP) in 0.3 wt. $\%$ and methyl ethyl ketone peroxide (MEKP) in $1.5 \mathrm{wt} . \%$ were used to accelerate and to polymerize the thermosetting polyester matrix resin, respectively. A bisphenolA-based thermoplastic polyester (ATLAC 363E) with fumerate groups in the backbone with a melting temperature of $60{ }^{\circ} \mathrm{C}$ was employed as preforming binder. Fabric preforms containing of 25 layers of stacks with 3 and $6 \mathrm{wt} . \%$ of thermoplastic polyester binder were obtained by application of heat and pressure as described in detail elsewhere $[1,5]$. The preform thickness with and without binder was measured using a micrometer. The thickness values of the preforms with and without binder were the average of the measurements from at least 10 different points and assumed as constant for all samples prior to VARTM processing. The preforms were measured to have average thickness of $21.80,13.15$, and $16.60 \mathrm{~mm}$ for 0,3 and $6 \mathrm{wt} . \%$ of binder, respectively. The composite panels were manufactured by VARTM method using the fabric preforms with and without binder under a vacuum pressure of $10 \mathrm{~Pa}$. After curing of the resin at room temperature, the cured panels were post-cured at $110^{\circ} \mathrm{C}$ for $2 \mathrm{~h}$. The fiber volume fraction values of each composite specimens subjected to compression test were measured based on the matrix burn-out technique. Compression test method according to ASTM D 695-M was used to measure the ply-lay up compressive strength of the composite specimens with and without binder.

\section{Artificial neural networks}

ANNs are basically a data-driven black-box model capable of solving highly non-linear complex problems. They have the ability to capture the relationship between input and output variables from given patterns (historical data or measured data on input and output variables of the system of the concern) and this enables them to solve large-scale complex problems. The network learns basically by finding the optimal network-connection-weights that would generate an output vector as close as possible to the target values of the output vector, with the selected accuracy. The optimal network-connection-weights are found by minimising the error function. The optimal network-connection-weights store the relationship between the input and output variables of the system from the given patterns.

In this study, three-layer feed forward artificial neural network (ANN) model having three input neurons, one output neuron and two hidden neurons was used. The corresponding model illustration is given in Fig. 1. In a feed forward network, the input quantities are first normalized to a range of $0.1-0.9$ with the following equation.

$X_{i}=0.1+0.8^{*}\left(X_{i}-X_{\min i}\right) /\left(X_{\max i}-X_{\min i}\right)$

where $X_{\max i}$ and $X_{\min i}$ are the maximum and minimum values of the $i$ th node in the input layer for all the feed data vectors, respectively. The weights were assigned a random value between -1 and 1 . Before its application to any problem, the network is first trained, whereby the difference between the target output and the calculated model output at each output neuron is minimized by adjusting the weights and biases through some training algorithm. During training, a neuron receives inputs from a previous layer, weights each input with a prearranged value, and combines these weighted inputs. The combination of weighted inputs is represented as

$n e t_{j}=\sum x_{i} v_{i j}$

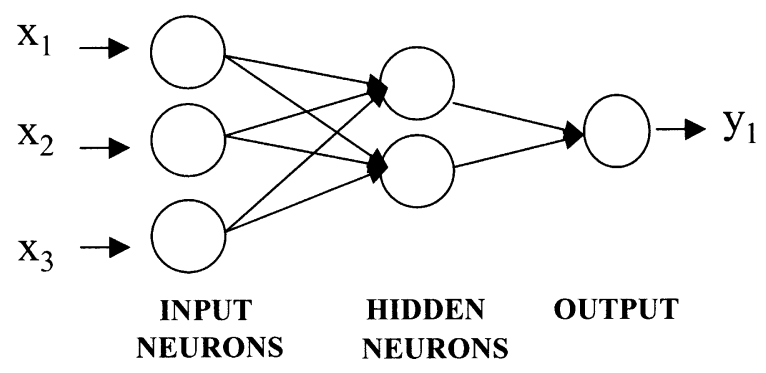

Fig. 1. Illustration of three layer feed forward ANN model. 
where $n e t_{j}$ is the summation of the weighted input for the $j$ th neuron, $x_{i}$ is the input from the $i$ th neuron to the $j$ th neuron, and $v_{i j}$ is the weight from the $i$ th neuron in the previous layer to the $j$ th neuron in the current layer.

The $n e t_{j}$ is passed through a transfer function to determine the level of activation. If the activation of a neuron strong enough, it produces an output that is sent as an input to the other neurons in the successive layer. In the present study, a sigmoid function given in Eq. (3) is employed as an activation function in the training of the network.

$f\left(n e t_{j}\right)=\frac{1}{1+\mathrm{e}^{-n e t_{j}}}$

The learning of ANNs was accomplished by a backpropagation algorithm where the information is processed in the forward direction from the input layer to the hidden layer and then to the output layer.

The objective of a back propagation network is, by minimizing a predetermined error function, to
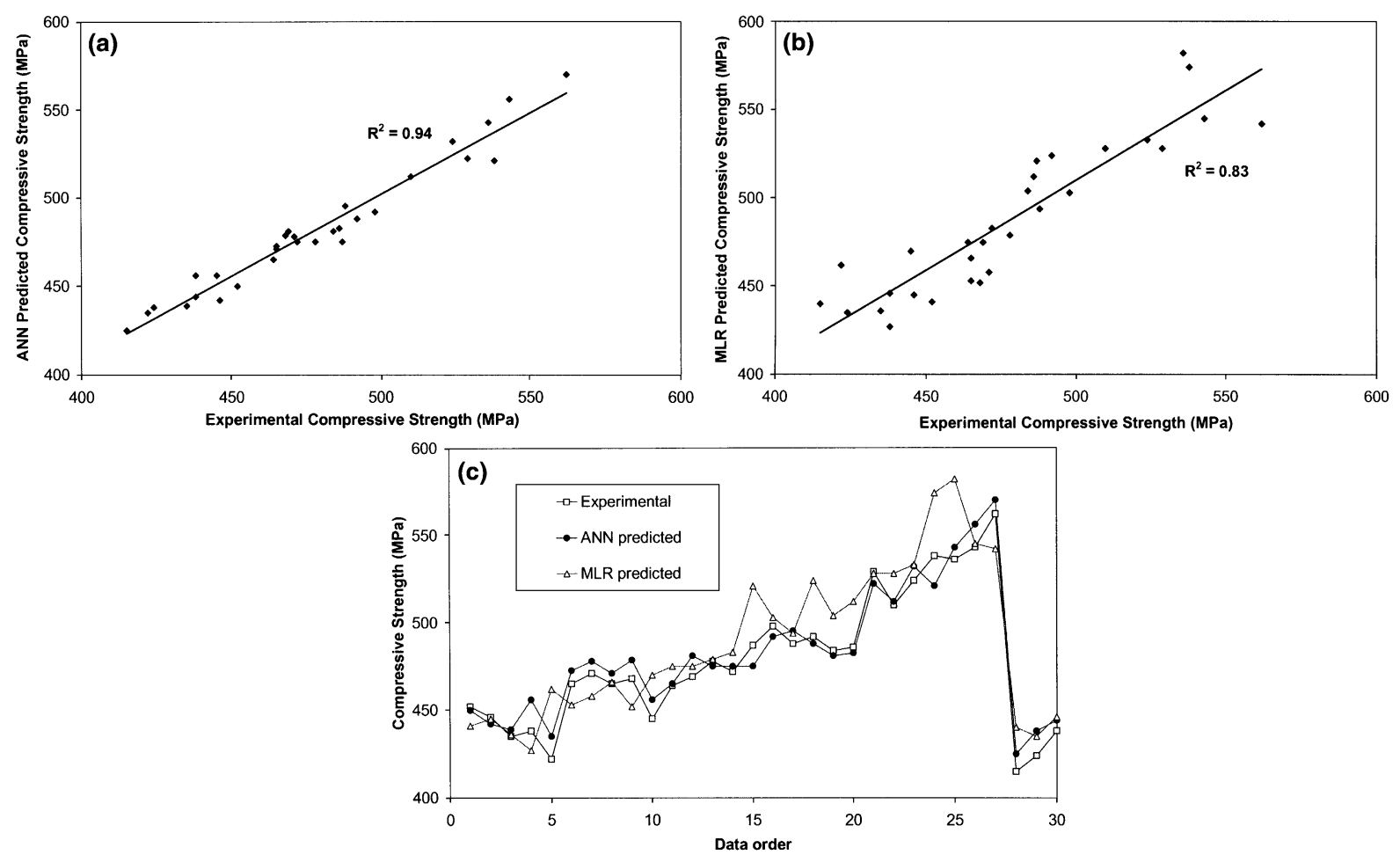

Fig. 2. Results of training: (a) ANN model prediction, (b) MLR prediction and (c) the model trend with data order.
Table 1

Input $(X)$ and output $(Y)$ parameters of ANN [5]

\begin{tabular}{llll}
\hline Code & Parameter & Minimum & Maximum \\
\hline$x_{1}$ & $\begin{array}{l}\text { Thermoplastic binder } \\
\text { amount (wt.\%) }\end{array}$ & 0 & 6 \\
$x_{2}$ & $\begin{array}{l}\text { Fiber preform } \\
\text { thickness (mm) }\end{array}$ & 13.15 & 21.80 \\
$x_{3}$ & $\begin{array}{l}\text { Composite fiber } \\
\text { volume fraction (-) }\end{array}$ & 0.34 & 0.57 \\
$Y$ & $\begin{array}{l}\text { Ply-lay up compressive } \\
\text { strength (MPa) }\end{array}$ & 415 & 574 \\
\hline
\end{tabular}

find the optimal weights that would generate an output vector $Y=(y 1, y 2, \ldots y p)$ as close as possible target values of output vector $T=(t 1, t 2, t 3 \ldots t p)$ with a selected accuracy. A predetermined error function has the following form:

$E=\sum_{p} \sum_{p}\left(y_{i}-t_{i}\right)^{2}$

where $y_{i}$ is the component of an ANN output vector $\mathbf{Y}, t_{i}$, is the component of a target output vec-

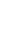


tor $\mathbf{T}, p$ is the number of output neurons and $P$ is the number of training patterns.

The least square error method, along with a generalized delta rule, is used to optimize the network weights. The gradient descent method with momentum term, along with the chain rule of derivatives, is employed to modify network weights as

$V_{i j}(n)=-\delta \frac{\partial E}{\partial V_{i j}}+\alpha V_{i j}(n-1)$

where $\delta$ is the learning rate that is used to increase the chance of avoiding the training process being trapped in a local minima instead of a global minima. The ANN is coded using $\mathrm{C}++$.

\section{Results and discussion}

ANNs having three input and one output neurons were used to model the ply-lay up compres- sive strength of the composites. The number of hidden neurons was taken two as a result of trying different number of neurons. The input variables used in the models were the amount of thermoplastic binder, initial fiber preform thickness prior to VARTM process and composite fiber volume fraction. Ply-lay up compressive strength of the composites was used as the output for the neural networks. The input and output parameters are given in Table 1 with their minimum and maximum values. The ANNs algorithm written in $\mathrm{C}++$ was trained and tested with sets of experimental data consisting of input and output values.

During the neural network scheme our input parameters are presented to the input layer nodes, input layer nodes are only used for input presentation. Then each input parameters are multiplied by the corresponding weight parameter. After the multiplication, results are summed and inserted to the connected middle layer node. Presented results are evaluated with the sigmoid activation
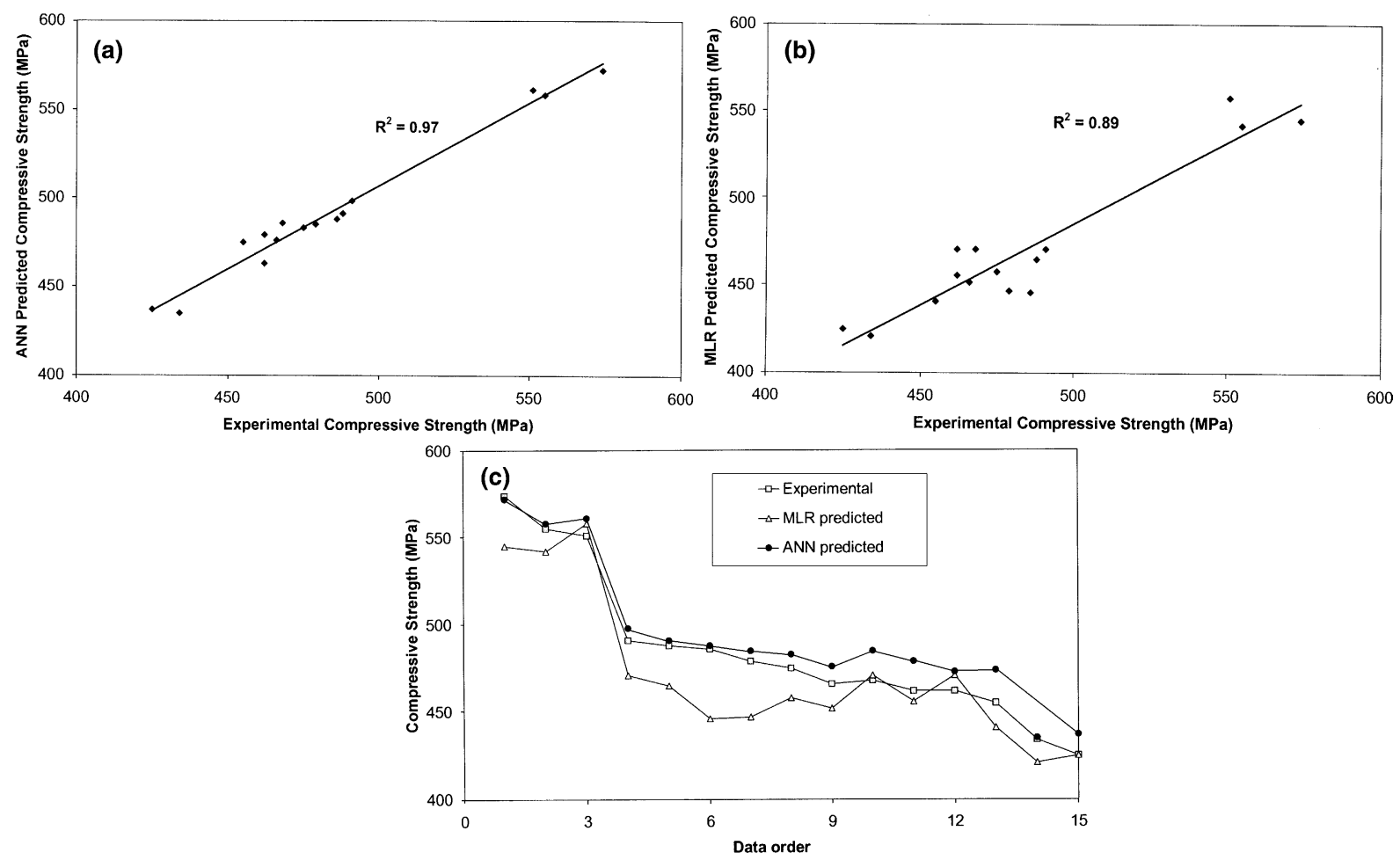

Fig. 3. Results of testing: (a) ANN model prediction, (b) MLR prediction and (c) the model trend with data order. 
function in the each middle layer neuron. Obtained results are multiplied again with the corresponding output weight function to present through the output layer. At this time, output layer is constructed with one linear output node (which represents our compressive strength estimation within the present study). Output layer node gives us our network's output. The obtained result is compared with the known target value to calculate the error value. Obtained error value's gradient with respect to the corresponding weight value lead us to the optimal solution by finding the optimal weights.

Bias term was not used during modeling but a momentum term was used to help to obtain faster convergence during iterations. This provided the iteration process not to get stuck in local minima, but rapidly reached the desired global minima. There were a total of 45 data sets that were divided into two groups for training and testing, each containing 30 and 15 sets, respectively. The program was instructed to run for 100,000 iterations and the optimal weights were calculated with an average percentage training error of $3.28 \%$. In addition, MLR model with the same input data was also employed to evaluate the results with ANN model. Fig. 2(a) and (b) shows the training of the ANN model and the MLR predictions, respectively. ANN model predicted the experimental
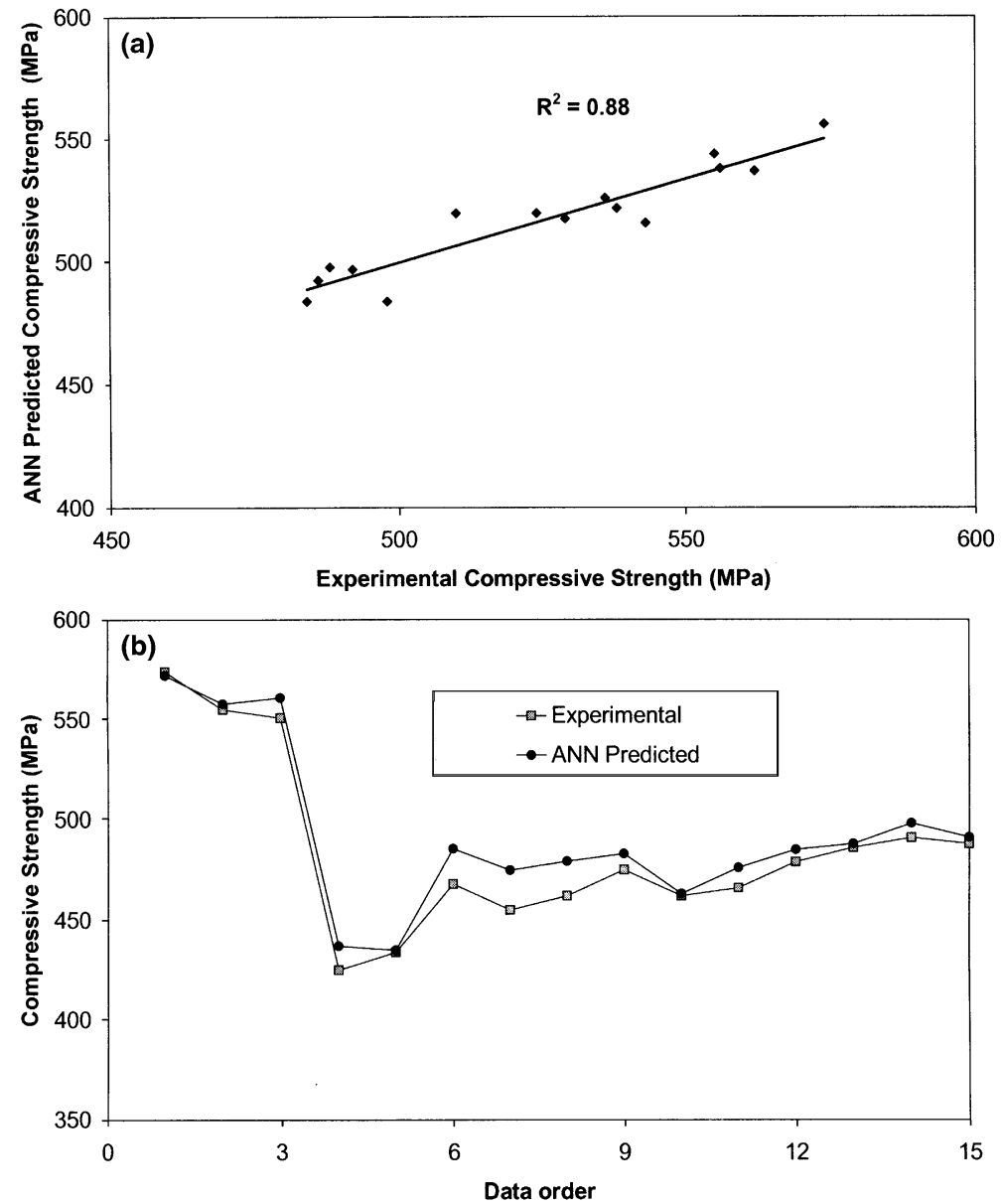

Fig. 4. Prediction of the ply-lay up compressive strength of the composites with 3 wt.\% of binder for sensitivity analysis. (a) ANN prediction and (b) trend with experimental data order. 
strength measurements with the correlation coefficient $\left(R^{2}\right)$ of 0.94 , showing better agreement than those of MLR with the correlation coefficient $\left(R^{2}\right)$ of 0.83 . Thus, as seen in Fig. 2(c), the values with the ANN model prediction were able to follow the trend better, as compared those with MLR model prediction. Fig. 3(a) and (b) shows the correlation coefficients $\left(R^{2}\right)$ of 0.97 and 0.81 for the ANN testing set and MLR model, respectively. In Fig. 3(c), ANN model and MLR predictions with experimental data order is given. Furthermore, the sensitivity analysis was performed by feeding ply-lay up compressive strength of the composites with 0 and 6 wt. $\%$ of binder as input into the developed ANN model to predict the compressive strength for the composites with 3 wt. $\%$ of binder as output. Fig. 4(a) and (b) shows the sensitivity analysis results. The correlation coefficient $\left(R^{2}\right)$ was 0.88 (Fig. 4(a)). The exact values of the measured strengths could not be obtained from the model as seen in (Fig. 4(b)). This was to be expected because the model was conservative and needed more training data to learn the extremes.

\section{Conclusion}

An ANN approach was successfully applied to predict the ply-lay up compressive strength of the composites by considering the effects of the thermoplastic binder amount, fiber preform thickness prior to VARTM process and the composite fiber volume fraction. The comparison of the ANN predictions with the experimental measurements was satisfactory. Moreover, the predicted values of ANN model were compared with those of a multi-linear regression (MLR) model. It was found that ANN had better predictions of the experimental compressive strength values than those with MLR. Furthermore, the sensitivity analysis was done to evaluate the performance of the ANN model. The results were found to be consistent with the experimental observations, but to have a lower correlation coefficient $\left(R^{2}\right)$. This indicates that the number of training dataset is critical for the ANN model sensitivity and predictive quality. As a result, it may be concluded that the ANN is a useful tool in characterizing the effects of some critical material parameters on the properties of the polymer composites if especially a sufficient amount of experimental data is obtained.

\section{References}

[1] M. Tanoglu, A.T. Seyhan, Investigating the effect of a preforming binder on the mechanical properties and ballistic performance of the E-glass reinforced polyester composites, International Journal of Adhesion and Adhesives 23 (2003) $1-8$.

[2] R.A. Saunders, C. Lekakou, M.G. Bader, Compression in the processing of polymer composites 1 . A mechanical and microstructural study for different glass fabrics and resins, Composites Science and Technology 59 (1999) 983-993.

[3] B. Chen, T.W. Chou, Compaction of woven fabric preforms in liquid composite molding processes: single layer deformation, Composites Science and Technology 59 (1999) 1519-1526.

[4] J.A. Acheson, P. Simacek, S.G. Advani, The implications of fiber compaction and saturation on fully coupled VARTM simulation, Composites Part A 35 (2004) 159-169.

[5] M. Tanoglu, A.T. Seyhan, Compressive properties of the E-glass reinforced polyester composites tailored with a thermoplastic preforming binder, Material Science and Engineering A 363 (2003) 335-344.

[6] Z. Zhang, N.M. Barkoula, J.K. Kocsis, K. Friedrich, Artificial neural network predictions on erosive wear of polymers, Wear 255 (2003) 708-713.

[7] F. İnal, G. Tayfur, T.R. Melton, S.M. Senkan, Experimental and artificial neural network modeling study on soot formation in premixed hydrocarbon flames, Fuel 82 (2003) 1477-1490.

[8] Z. Zhang, P. Klein, K. Friedrich, Dynamic mechanical properties of PTFE based short carbon fiber reinforced composites: experiment and artificial neural network prediction, Composites Science and Technology 62 (2002) 1001-1009.

[9] S. Akkurt, S. Özdemir, G. Tayfur, B. Akyol, The use of GA-ANNs in the modeling of compressive strength of cement mortar, Cement and Concrete Research 33 (2003) 973-979. 